\title{
Cecum Carcinoma
}

National Cancer Institute

\section{Source}

National Cancer Institute. Cecum Carcinoma. NCI Thesaurus. Code C3491.

A malignant epithelial neoplasm that arises from the cecum and invades through the muscularis mucosa into the submucosa. The vast majority are adenocarcinomas. 